\title{
The Internet of Things - Emerging Technologies, Challenges and Applications
}

\author{
Sanket Thakare \\ Software Developer \\ ebuild Softwares and Solutions \\ Mumbai- 400051, India
}

\author{
Ashwini Patil \\ Assistant Professor \\ Thakur College of Engineering \\ and Technology \\ Mumbai- 400101, India
}

\author{
Ashraf Siddiqui \\ Lecturer \\ Theem College of Engineering \\ Boisar- 401501, India
}

\begin{abstract}
In this paper, we describe the Internet of Things (IoT) which is a paradigm where things can have identifying, sensing, and processing features that will allow them to communicate with other devices over the Internet to perform the task. There are many challenges with software, hardware, and security in actual implementation of IoT. The traditional networking protocol and security services are not directly applied on IoT because of different communication stacks and various networking standards. So, there is need to find an efficient solution which deals with the all the challenges in an environment of IoT. This paper presents various research challenges with their respective solutions by examining previous literature and identifying current trends. It also discusses future directions in the field of IoT.
\end{abstract}

\section{Keywords}

IoT, RFID, Sensor Networks, Sensors

\section{INTRODUCTION}

Nowadays, the Internet has become the most important part of people's life. Billions of people around the world connected to each other with Internet by sending and receiving emails, using social networking applications, playing online games and many more things. This can be called the "Internet of People". The technologies like Sensor Networks and RFID tags are evolving with rapid development in the field of Internet technologies. With a combination of these two technologies, i.e. the Internet and Sensor Networks leads to a new vision of direct machine to machine communication over the Internet. This can be called "Internet of Things".

There is no specific definition of IoT, however, the idea is to make the everyday objects capable of identifying, sensing, networking over the internet to perform some task. The idea of direct communication between machines to machine exists from many years like client and server machine communication over the Internet. But the IoT represent the idea of attaching technology with devices like home appliances, cars, street light and integration of this devices with a network. It also can make capable of identifying the specific device and accessing it over the Internet with RFID technology. The motivation for the research in the field of IoT is to create a Smart city. The main goal is to make a better world for human being where every device around can understand the situation and perform an action without any explicit instruction. Implementation of IoT concept came with different technological and social challenges. The IoT device has low resources in terms of computation and battery capacity. So, the solution for implementation of IoT should be efficient in terms of resource utilization, secure and interoperable in an interconnected network. The main objective of this paper is to understand challenges in the implementation of Iot, currently available solutions and applications of IoT.

The rest of the paper is organized as follows. Section 2 presents the definition and vision of IoT. Section 3 describes the evolution of Internet of Things. Section 4 describes the key elements that play an important role in IoT. Section 5 describes a layered architecture framework for IoT and also issues involved in different layers. Section 6 presents different protocol designed for implementation of IoT. Section 7 identifies the challenges in the implementation of IoT in the real world. Section 8 presents some applications of IoT in various industry. Section 9 summarizes our study and conclude the paper.

\section{INTERNET OF THINGS}

The term IoT is an interconnected network of smart objects using the Internet, sensors, and RFID is able to transfer data without human interaction over the network. It consists of the (i) network which interconnects objects by using Internet technologies (ii) set of technologies such as Radio Frequency Identifications (RFIDs) and sensors [1]. RFID systems and sensor networks are performing the main role in the vision of IoT because of its maturity, low cost, and support from the business community. In a 2009 presentation, SAP Research's Stephan Haller defined the Internet of Things as "the world where physical objects are seamlessly integrated into the information network, and where the physical objects can become active participants in business processes"[2]. Many companies and research organization project a wide range of prognostication of IoT in next five years. Cisco estimates that IoT will consists of 50 billion devices connected to the Internet by 2020. However, Morgan Stanley projects there will be 75 billion networked devices by 2020. Huawei predicts 100 billion IoT connections by 2025 . The popular and simple example is the smart fridge which could tell you that it was out of milk. The internal camera in fridge monitor the milk container and send a text to a mobile phone if there was no milk left. IoT has a bigger vision than smart homes. It includes smart cities with smart traffic signals and smart beans send a signal to make it empty and industries in which everything has sensors from tracking part to monitor crops.

\section{EVOLUTION OF IOT}

In 1999, The British technology pioneer Kevin Ashton use the term "Internet of Things" first time to describe the system in which all devices with sensors in the real world connected to each other. The term "Internet of Things" is new to us but the concept of using devices to monitor and control exists from the decade. In the late 1970s, the systems that remotely monitor meters on the electrical grid used. [3] In 1990, the first internet toaster which turned on or off was presented in 
internet conferences. In 2001, other things came with IPbased like a soda machine at Carnegie Mellon University in the US and a coffee pot in the Trojan Room at the University of Cambridge in the UK [4]. The European Commission (EC) had made a lot of efforts in the field of IoT since 2006. In 2008, EC published a Staff Working Document to discuss policy issues in the governance of IoT. In 2009, IBM's CEO S. Palmisano proposed the concept of "Smart Planet" in which everyday item like the power grid, airports, railway station are equipped with sensors [5], [6]. Today, the IoT devices are still primarily things on the Internet that require more human interest and research. The evolution of the IoT is just starting to be realized.

\section{ELEMENTS OF IOT}

The elements of IoT play an important role in understanding the IoT functionality [7]. The following section describes different elements of IoT and their role.

\subsection{Identification}

Identification is one of the important element which is used to uniquely identify the device and provide required service to it in IoT network. The different Identification methods are used to identify IoT objects like as electronic product codes (EPC) and ubiquitous codes (uCode) [8]. IPv6 and IPv4 are used for addressing methods of IoT objects. The IPv6 header is compressed with 6LoWPAN compression mechanism that makes IPV6 efficient for low power wireless networks [9] [10].



Fig 1: Elements of IoT

\subsection{Sensing}

The IoT sensing is used to collect data from a different object in the network and send it to the database.

The collected data is used to take respective action. Various IoT sensors are available in the market such as smart sensors, actuators, etc. For example, a company like Smart-Things used smart hubs and mobile application to control home appliances and make a home as smart home. In a smart home, people can monitor and control thousands of devices with their smartphone [11], [12]. The devices like Raspberry PI, BeagleBone Black are integrated with sensors and used as IoT devices and provide required data to the customer.

\subsection{Communication}

IoT communication element is used to connect different IoT objects to each other. IoT devices have low power and noisy communication link. RFID is the first technology used for Machine to machine communication. It uses RFID tags and Reader for communication. RFID tag is a label attach to the specific device. RFID readers send a query signal to RFID tag and receives signal send by tag. Then, it is passed to database which is processed by connected processing center and the object is identified in a particular range. Wifi is another communication technology used to exchange data in $100 \mathrm{~m}$ range [13]. It can communicate and exchange data without using a router. Bluetooth is also a communication technology used to exchange data between devices over a short distance. It uses short-wavelength radio to minimize power consumption [14]. The Bluetooth special interest group (SIG) introduce Bluetooth 4.1 which required low energy and provides as high-speed and IP connectivity [15].

\subsection{Computation}

Hardware like microcontrollers, microprocessors, and software applications are used for the computation of IoT. Various Hardware platform has been developed for IoT like Arduino,Raspberry PI,Gadgeteer, BeagleBone, Cubieboard, etc. Various Software platforms have been developed like RTOS operating System. TinyOS [16], LiteOS [17] and RTOS [18] are different lightweight OS that are designed for IoT environments.

\section{ARCHITECTURE OF IOT}

The architecture of IoT is based on different layers. It contains data layers at the bottom to application layers at the top. The layered architecture of IoT is designed in such a way that it can satisfy the need of different industry, institutes, and governments etc. Figure 2 describes the layered architecture of IoT [19]. The layers in IoT architecture divided into two parts by internet layer. Top two layer are used for data utilization in the application and two bottom layers are used for data capturing. The functionality of different layers is described below.

\subsection{Edge Layer}

Edge Layer is the lowest layer in the layered architecture of IoT. This layer consists of different elements like IoT devices, sensor, and RFID tags. These elements give information about identification, processing, and communication.

\subsection{Access gateway layer}

This layer handles the data in the IoT environment. It performs an operation like publishing route message and in some cases perform cross communication. 


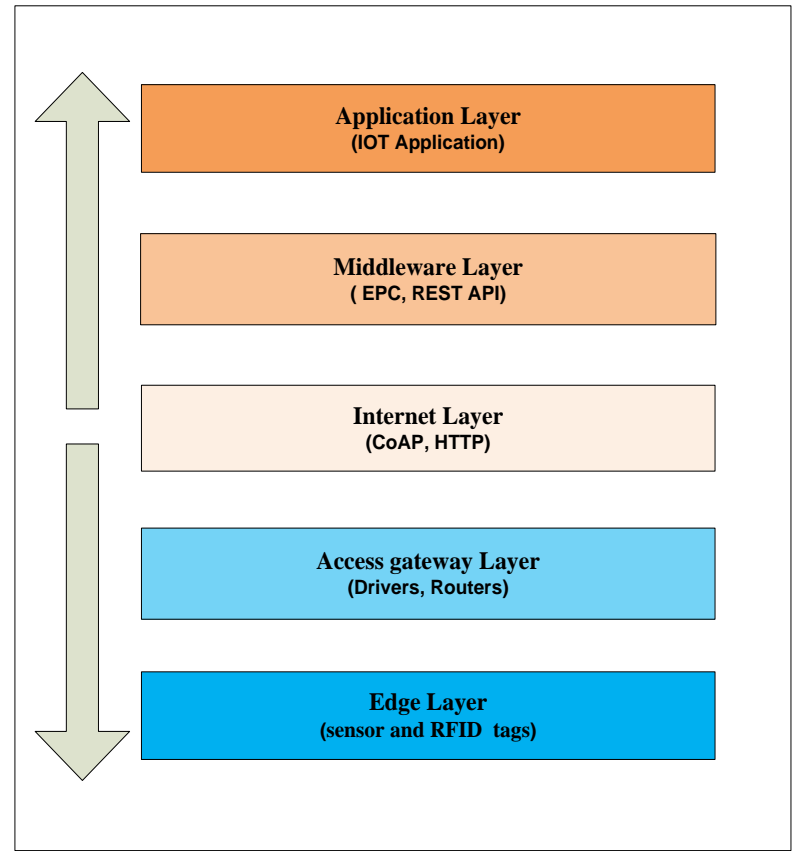

Fig 1: IoT Layered Architecture

\subsection{Middleware layer}

This is one of the important layers in IoT layered architecture which acts as an interface between hardware layer and application layer [20]. It is mainly used for device management and perform functions like data filtering, semantic analysis, information discovery.

\subsection{Application layer:}

This is the top most layer in the IoT layered architecture. Its main function is to deliver different applications to users [21]. Applications can be from a different category like manufacturing, industries, food, environment, etc.

\section{IOT PROTOCOLS}

In IoT, identifying the protocol design for interconnection of the sensor device is a main challenging task. Institute of Electrical and Electronics Engineers (IEEE) and the Internet Engineering Task Force (IETF) are designing new communications and security protocols that will play a fundamental role in the implementation of IoT applications. While designing protocol for IoT, low-energy sensing devices and low-rate wireless communications are the main constraint. In IoT, device gathered the information on the basis of sensors and sends the data to the server. The server analyzes it and shares the data by sending it back to devices or to people. The following section describes a various protocol for that can be used in IoT Framework [22].

\subsection{MQTT}

MQTT is the Message Queue Telemetry Transport protocol. It is used for collecting data from IoT devices and send it to Servers. Its main purpose is telemetry or remote monitoring. It is used to monitor and control a large network of small IoT devices from the cloud. It is simple and also offering few control options. In MQTT, real time is measured in seconds. It is used for an application for monitoring an oil pipeline for leaks, power usage monitoring, lighting control, and even intelligent gardening. It collects data from these devices and share that data with servers and make it available.

\subsection{XMPP}

XMPP is an Extensible Messaging and Presence Protocol. It was developed for connecting people with instant messaging (IM) via text messages. It uses XML text format as its native type for the communication. In IoT, it provides an easy way to address a device. In XMPP, real time is measured in seconds. It provides a way to connect home thermostat to web server and access it through the phone. It is an efficient solution for consumer-oriented IoT applications because of addressing, security, and scalability features.

\subsection{DDS:}

DDS is Data Distribution Service (DDS). It is used to connect devices to other devices and distributes data to other devices. In IoT, the device needs to communicate with another device. TCP is the reliable and simple solution for connecting devices. But, it is very restrictive. DDS offers detailed qualityof-service (QoS) control, multicast, configurable reliability, and pervasive redundancy. It has efficient ways to filter data and send selected data to thousands of simultaneous destinations. There are also lightweight versions of DDS for some small IoT devices. DDS is used with High-performance integrated device system. It provides flexibility, reliability, and speed to build complex, real-time applications. It is able to efficiently deliver millions of messages per second to many simultaneous receivers. In DDS, real time is measured in microseconds. It is used for an application like military systems, wind farms, hospital integration, medical imaging, and asset-tracking systems.

\subsection{AMQP}

AMQP is the Advanced Message Queuing Protocol. Its main goal is not losing messages during communication. It uses TCP for communication from queues to subscribers which provides reliable point-to-point connection and endpoint also send acknowledge of acceptance of each message. It tracks all messages and ensures that each message is delivered to intended endpoint. It is mostly used for business messaging. In the IoT, it is appropriate for server-based analysis functions.

\section{CHALLENGES OF IOT}

IoT faces a lot of challenges in terms of both technical and social issues. These challenges must be overcome in order to the actual implementation of IoT in the real world [23]. Challenges are classified as follows:

\subsection{Security}

IoT devices are small wireless devices that would be placed in public places. Wireless communication is made secure through encryption technique. But the IoT devices are very small and not powerful enough to support encryption methods. There is need to modify encryption algorithm in order to support IoT devices. The algorithm should efficient and less energy consuming.

\subsection{Privacy}

In IoT, the different devices are traceable through the interconnected network, it creates threats to personal and private data. There should be assurance that issue of data ownership addressed in IoT in order to avoid data fell into the wrong hand. The data owner must be ensured that data will not be used without her/his permission. The privacy policy is the solution to keep data secure in IoT environment. Whenever other user came into contact for reading data, it can check the other's privacy policy before communicating. 


\subsection{Standards and interoperability:}

To implement any new technology in the market, it should follow the specific standard. If devices are manufactured by the different manufacturers without following the specific standard, it will be very difficult to make devices interoperable. It may require some extra gateway to translate from one standard to another. The European Commission is working on a technological standard to support IoT.

\subsection{Scalability:}

Computer Network researchers always have a question of scale. Today, Internet routing system interconnects more than 3 billion people and more than 50 thousand separately administered networks. Networking Community handles this requirement by designing routing protocols and routing architectures. But, today's routing protocols are designed for a system with reasonable computation and memory resources. IoT devices are small and low with computation power. So, there is a need to design future network protocol and network architectures for low computation and memory devices.

\subsection{Low power communication:}

Many IoT devices are small in size and do not have the continuous power source. A device computation depends on battery size and cost of the device. Many IoT devices work as a single, limited purpose which could have customized network interfaces, operating systems, and programming models that make the most efficient use of limited computation, network, and energy resources. Research in these areas involves the interdisciplinary collaboration of signal processing and wireless communication as well as computer architectures and operating systems.

\subsection{Security threats from ubiquitous devices:}

If a desktop computer got infected with the virus, it can be a reboot or can remove viruses by running some antivirus and problem may get solved. But, if IoT devices got affected with viruses, then it will be very difficult to know which device has been compromised and how to restore system security. So, there is need of a systematic method for restoring IoT System and also need a tool to isolate and correct compromised components within IoT network.

\subsection{Debugging self-diagnosing, and automatic repair:}

Debugging and updating the code running on IoT devices is a very challenging task in IoT world. It is very difficult to do interactive debugging on IoT devices because of limited bandwidth. It is also difficult to store detailed logs of devices because of its low power and memory constrained. Devices need to have software system which can learn from errors and automatically repair themselves.

\section{APPLICATIONS OF IOT}

With IoT number of applications can be build and very few applications are developed currently. There will be various applications in future like smarter home, smarter transportation, smarter hospitals, smarter factories, etc [24] [25]. In the following section, different IoT applications are discussed.

\subsection{Automotive industry:}

Today, vehicles become advanced with having advanced sensors, actuators. In the automotive industry, smart things can be used to monitor and get a report from air pressure in tires to a different part of vehicle status. RFID technology has already been used increase quality control and improve customer services. The device attached to vehicle part contains information about the part like manufacturing date, serial number, product code and some also have location information according to vehicle movement. Vehicle-tovehicle (V2V) and Vehicle-to-infrastructure (V2I) communications is an advanced Intelligent Transportation Systems (ITS) which can be used in smart traffic control and vehicle safety service. The IoT devices can be integrated with the Intelligent Transportation Systems for a better result.

\subsection{Pharmaceutical industry}

In IOT, the smart label can be attached to drugs and track them through supply chain management and monitor the status of drugs. Suppose, medicine required cold storage and cold storage has not been provided during the transportation. The smart label on drugs will discard that medicine. This way medicine can keep the supply chain free of fraudsters. The smart label can also help the patient by sending information regarding dosage and expiry date. It can remind patient to take their at appropriate interval and patient compliance can be the monitor.

\subsection{Manufacturing industry}

In the manufacturing industry, the production process can be optimized with the use of smart devices or unique identifiers. It can interact with an intelligent supporting network and monitor the process from production to disposal. Status of production machine can get by tagging item and container. Self-organizing and intelligent manufacturing solutions can be derived with identifiable items in manufacturing industry.

\subsection{Media, entertainment industry}

In the media industry, IOT device enables news gathering based on user location. The news can be gathered by sending queries to multimedia devices present at a certain location. Communication tags can be attached to the poster to give detail information to the user. It transfers requesting the user to a URI address that has detailed information.

\subsection{Agriculture and breeding}

In agriculture, IOT devices can be used to trace agricultural animal and their movement. In many countries, government issues subsidy based on a number of animal in farms. But, it's very difficult to determine the exact number. With the help of IOT devices, this type of frauds can be minimized. With the help of IOT, a farmer can deliver crops directly to small as well as the wide region. This will change the supply chain management that is handle by large companies.

\subsection{Supply chain management}

IOT can provide many advantages in the field of supply chain management operations. The retailer can track the item or shelves which are equipped with RFID tag. It will be easy to monitor the stock, tracing out of stock items. It can be seen that $3.9 \%$ of sales loss happens because of empty shelves delivered to the customer. With IOT, Shelves with sensors helps to avoid this type of losses. Supply chain management is already supported by various IT solutions. In traditional supply chain management, information is passed one's direct downstream partner and not shared among the whole chain. In IOT, devices with RFID records all details like manufacturing information, production date, expiry date, warranty period and makes supply chain management more efficient. 


\section{CONCLUSION}

The Internet of Things (IoT) is one of the emerging research topics in which many researchers are working. The main goal of IoT is to provide quality of life to all human beings by connecting all objects in the environment with technologies. The IoT will bring automation to all things around us. Cloud service providers like AWS (Amazon Web Services), Microsoft Azure, Google also started providing a platform for IoT deployment. In IoT, Machine to Machine communication will increase data massively. It also leads to the evolution of Big Data. But, the protection and privacy of user's data are one of the key challenges in the IoT. This paper described an overview of IoT architecture, its element and issues and challenges of IoT for researchers and developers. Further, it also describes applications of IoT in the real world. The current technologies making the idea of IoT to reality, still many challenges exist for deployment of IoT application in real world. In future, networking and communication research have an opportunity to address this challenges.

\section{REFERENCES}

[1] C. Perera, A. Zaslavsky, P. Christen, and D. Georgakopoulos, "Context-Aware Computing for The Internet of Things: A Survey" IEEE Communications Surveys \& Tutorials, 2013, pp. 1-41

[2] The Internet of Things: A Seamless Network of Everyday Objects, July 31, 2013. Available from: URL: http://www.livescience.com/38562-internet-ofthings.html (accessed 7. 24. 15).

[3] "Machine to Machine." Wikipedia, the Free Encyclopedia, August 20, 2015. Available from: URL: https://en.wikipedia.org/wiki/Machine_to_machine (accessed 7. 27. 15).

[4] Stafford-Fraser, Quentin. "The Trojan Room Coffee Pot." N.p., May 1995. Web. 06 Sept. 2015. Available from:URL:http://www.cl.cam.ac.uk/coffee/qsf/ coffee.html (accessed 8.4.15).

[5] Commission Staff Working Document, Future Networks and the Internet - Early Challenges regarding the "Internet of Things", in COMMISSION OF THE EUROPEAN COMMUNITIES, 2008.

[6] IBM builds a smarter planet. Available from: URL: http://www.ibm.com/smarterplanet/us/en/.

[7] Ala Al-Fuqaha, Mohsen Guizani, Mehdi Mohammadi, Mohammed Aledhari, Moussa Ayyash, "Internet of Things: A Survey on Enabling Technologies, Protocols, and Applications", in IEEE Communications Surveys \& Tutorials, 2015.

[8] N. Koshizuka and K. Sakamura, "Ubiquitous ID: Standards for Ubiquitous Computing and the Internet of Things," Pervasive Computing, IEEE, vol. 9, pp. 98-101, 2010.

[9] N. Kushalnagar, G. Montenegro and C. Schumacher, "IPv6 over low-power wireless personal area networks (6LoWPANs): overview, assumptions, problem statement, and goals," RFC4919, August, vol. 10, 2007.

[10] G. Montenegro, N. Kushalnagar, J. Hui and D. Culler, "Transmission of IPv6 packets over IEEE 802.15. 4 networks," Internet Proposed Standard RFC 4944, 2007.
[11] (Sept. 2014). SmartThings | Home Automation, Home Security, and Peace of Mind. Available: http://www.smartthings.com.

[12] U. Rushden, "Belkin brings your home to your fingertips with WeMo Home Automation System," Press Room Belkin, 2012.

[13] E. Ferro and F. Potorti, "Bluetooth and Wi-Fi wireless protocols: a survey and a comparison," Wireless Communications, IEEE, vol. 12, pp. 12-26, 2005.

[14] P. McDermott-Wells, "What is Bluetooth?" Potentials, IEEE, vol. 23, pp. 33-35, 2005.

[15] Press Releases Detail: Bluetooth Technology, sept, 2014 Available from:URL: http://www.bluetooth.com/Pages/ Press- Releases-Detail.aspx?ItemID=197.(accessed 8. 5. 16).

[16] P. Levis, S. Madden, J. Polastre, R. Szewczyk, K. Whitehouse, A. Woo, D. Gay, J. Hill, M. Welsh, E. Brewer and D. Culler, "TinyOS: An operating system for sensor networks," in Ambient IntelligenceAnonymous Springer, 2005, pp. 115-148.

[17] Q. Cao, T. Abdelzaher, J. Stankovic and T. He, "The LiteOS operating system: Towards Unix-like abstractions for wireless sensor networks," in Information Processing in Sensor Networks, 2008. IPSN '08. International Conference On, 2008, pp. 233-244.

[18] E. Baccelli, O. Hahm, M. G nes, M. W hlisch and T. C. Schmidt, "RIOT OS: Towards an OS for the internet of things,"in Computer Communications Workshops (INFOCOM WKSHPS), 2013 IEEE Conference On, 2013, pp. 79-80.

[19] L. Atzori, A. Lera, and G. Morabito. The Internet of Things: A Survey. Computer Networks 54(15), 27872805. (2010).

[20] Debasis Bandyopadhyay, Jaydip Sen, "Internet of Things - Applications and Challenges in Technology and Standardization" in Wireless Personal Communications , Volume 58, Issue 1, pp. 49-69

[21] Miao Wu, Ting-lie Lu, Fei-Yang Ling, ling Sun, HuiYing $\mathrm{Du}$, "Research on the architecture of Internet of things," in advanced Computer Theory and Engineering (ICACTE), 2010 , pp. 484-487

[22] Understanding The Protocols behind the Internet Of Things. Available from:URL:http://electronicdesign.com /IoT/understanding-protocols-behind-internet-things (accessed 8.6. 16).

[23] Rajeev Alur, Emery Berger, Ann W. Drobnis, Limor Fix, Kevin Fu, Gregory D. Hager, Daniel Lopresti, Klara Nahrstedt, Elizabeth Mynatt, Shwetak Patel, Jennifer Rexford, John A. Stankovic, and Benjamin Zorn,"Systems Computing Challenges in the Internet of Things", in eprint arXiv, April 2016.

[24] K. Bing, L. Fu, Y. Zhuo, and L. Yanlei, "Design of an Internet of Things-based Smart Home System," $2^{\text {nd }}$ International Conference on Intelligent Control and Information Processing, 2011, pp. 921-924.

[25] "What we're driving at," Google Official Blog. It can be accessed at http://googleblog.blogspot.com/ 2010 /10/what-were-driving-at.html 